\title{
Neuroplasticidad: Ejercicios para retrasar los efectos de la Enfermedad de Alzheimer mediante Estimulación Cognitiva
}

\author{
Neuroplasticity: Exercises to delay the effects of Alzheimer's disease \\ through Cognitive Stimulation
}

Gabriela Castillo $^{1}$; Brayhan Fernández ${ }^{1}$; Diego Chamorro ${ }^{1}$

\section{RESUMEN}

La neuroplasticidad se refiere a la capacidad del sistema nervioso para cambiar su estructura y su funcionamiento a lo largo de su vida, como reacción a la diversidad del entorno permitiendo a las neuronas regenerarse tanto anatómica como funcionalmente y formar nuevas conexiones sinápticas. Este potencial adaptativo del sistema nervioso permite al cerebro reponerse a trastornos o lesiones, y puede reducir los efectos de alteraciones estructurales producidas por patologías como lo es la enfermedad del Alzheimer. La enfermedad de Alzheimer es una enfermedad cerebral que ocasiona problemas relacionados con la memoria, el pensamiento y el comportamiento. No es una parte normal del envejecimiento. El Alzheimer empeora con el tiempo aunque los síntomas pueden variar mucho. El primer problema que muchas personas notan es el olvido lo suficientemente grave como para afectar su capacidad para funcionar en el hogar o en el trabajo, o para disfrutar de pasatiempos permanentes. La estimulación cognitiva es un conjunto de procedimientos muy útiles para estimular los mecanismos de la neuroplasticidad y con ello detener el avance de la demencia, así como mejorar la calidad de vida del paciente y de su familia.

Palabras clave: Enfermedad de Alzheimer, Neuroplasticidad, Sistema Nervioso, Memoria, Estimulación Cognitiva.

Fecha de recepción: agosto 2020; fecha de aceptación: octubre 2020

${ }^{1}$ Universidad Privada María Serrana, Carrera de Psicología. Asunción, Paraguay

Autor de correspondencia: Diego Chamorro. Email: diegoangel.py@gmail.com 


\section{ABSTRACT}

Neuroplasticity refers to the ability of the nervous system to change its structure and functioning throughout its life, as a reaction to the diversity of the environment, it allows neurons to regenerate both anatomically and functionally and to form new synaptic connections. This adaptive potential of the nervous system allows the brain to recover from disorders or injuries, and can reduce the effects of structural alterations produced by pathologies such as Alzheimer's disease. Alzheimer's disease is a brain disease that causes problems related to memory, thinking, and behavior. It is not a normal part of aging. Alzheimer's gets worse over time. Although symptoms can vary greatly, the first problem many people notice is forgetfulness severe enough to affect their ability to function at home or work, or to enjoy permanent hobbies. Cognitive stimulation is a set of very useful procedures to stimulate the mechanisms of neuroplasticity and thereby stop the progression of dementia, as well as improve the quality of life of the patient and their family.

Keywords: Alzheimer's Disease, Neuroplasticity, Nervous System, Memory, Cognitive Stimulation. 


\section{INTRODUCCIÓN}

"La neuroplasticidad o plasticidad cerebral es la potencialidad del sistema nervioso de modificarse para formar conexiones nerviosas en respuesta a la información nueva, la estimulación sensorial, el desarrollo, la disfunción o el daño" (Louder, 2018). El termino Plasticidad fue introducido en 1890 por el psicólogo William James para describir la naturaleza modificable del comportamiento humano.

En paralelo a los procesos que causan involución en el sistema nervioso, en el envejecimiento cerebral se producen mecanismos de plasticidad compensatoria que evitan que el deterioro cognitivo progrese más activamente en el cerebro de los ancianos. Cuando se inicia el proceso de atrofia cortical, las neuronas que no han resultado afectadas muestran una proliferación axónica y dendrítica más activa para compensar el déficit, especialmente en áreas cognitivas importantes para la consolidación del aprendizaje como el hipocampo. La pérdida de arborizaciones dendríticas en determinadas neuronas a partir de la vejez está acompañada por el incremento del árbol dendrítico de las neuronas que permanecen vivas, en compensación paralela al proceso de muerte neuronal (Kramer, 1999).

El envejecimiento es un proceso progresivo no imputable a las enfermedades, que afecta a todos los sistemas orgánicos produciendo cambios metabólicos y fisiológicos con el paso del tiempo. También se puede definir como la pérdida de capacidad adaptativa a cualquier cambio necesario para desarrollar la vida de un modo normal (Horton, 1997).

El ejercicio físico moderado realizado de modo continuado resulta muy beneficioso para el mantenimiento funcional del organismo en cualquier edad. Además, la actividad física moderada también me- jora las funciones cognitivas, aumentando los niveles de neurotrofinas, así como el número de neuronas en el hipocampo. Estos hechos también producen un efecto beneficioso en enfermedades psiquiátricas $\mathrm{y}$ neurodegenerativas. Este trabajo tiene como objetivos analizar los conceptos y el funcionamiento de la neuroplasticidad y el tratamiento de la demencia por Enfermedad de Alzeimer; describir los ejercicios que estimulen la neuroplasticidad y que contribuyan a la recuperación de enfermedades como del Alzheimer. Fue una nvestigación documental con utilización de la técnica de análisis de contenido considerando como categorías de análisis el tratamiento de la demencia por Enfermedad de Alzeimer, ejercicios de estimulación de neuroplasticidad, recuperación de enfermedades como del Alzheimer. Se recurrió a la utilización de los descriptores: Enfermedad de Alzheimer, neuroplasticidad, sistema nervioso, memoria, estimulación cognitiva.

\section{Neuroplasticidad}

El concepto de plasticidad neural (o neuroplasticidad) surge como alternativa que activa el cerebro humano para combatir este tipo de demencia a la que se ve sometido. En este sentido, el sistema nervioso posee una gran capacidad plástica, que le permite recuperarse de las lesiones que pueda sufrir en un momento determinado. Es la propiedad que tienen las células nerviosas de reorganizar sus conexiones y de modificar los mecanismos implicados en su comunicación con otras células. Estos cambios pueden traer como consecuencia la modificación del funcionamiento de los circuitos neurales y la forma como se relacionan los sistemas moda-les en que se organiza el cerebro. Este proceso sucede durante y después de su maduración en fases como la memoria y el aprendizaje. Actualmente se admite que las redes neuronales que componen el sistema nervioso 
de los mamíferos permanecen plásticas, modificables, a lo largo del curso entero de la vida de estos organismos. Dicha plasticidad constituye una de sus adaptaciones más importantes. Todo ello englobado bajo la denominación de plasticidad neural. (Nieto Sampedro, 2003)

De igual modo, el grado de recuperación depende de muchos factores, que incluyen la edad, el área del cerebro comprometida, la cantidad de tejido dañado, la extensión de la lesión, la rapidez con la que se pro-duce el daño, los programas de rehabilitación y los factores ambientales y psicosociales (Hernández Muela S, 2004)

Demencias. - Concepto de Demencias

La demencia es un deterioro adquirido, crónico y generalizado de las funciones cognitivas, que afecta a dos o más áreas: memoria, capacidad para resolver problemas, realización de actividades perceptivo-motrices, utilización de habilidades de la vida diaria y control emocional. El problema está causado por lesiones cerebrales, sin que inicialmente exista perturbación del nivel de conciencia. La severidad del déficit cognitivo en la demencia interfiere el normal funcionamiento familiar, social y ocupacional del sujeto. (Peña, 2019)

Trastorno neurodegenerativo mayor o leve debido a la enfermedad de Alzheimer.

La enfermedad de Alzheimer es una enfermedad cerebral que ocasiona problemas relacionados con la memoria, el pensamiento y el comportamiento. No es una parte normal del envejecimiento. El Alzheimer empeora con el tiempo. Aunque los síntomas pueden variar mucho, el primer problema que muchas personas notan es el olvido lo suficientemente grave como para afectar su capacidad para funcionar en el hogar o en el trabajo, o para disfrutar de pasatiempos permanentes.

La enfermedad puede causar que una persona se confunda, se pierda en lugares conocidos, extravíe las cosas o tenga problemas con el lenguaje. Puede ser fácil de explicar el comportamiento inusual como parte del envejecimiento normal, especialmente cuando alguien parece físicamente saludable. (Association, 2016)

La Enfermedad de Alzheimer constituye la modalidad de demencia más frecuente, ya que se estima que el $70-75 \%$ de los casos corresponde a esta patología, seguida de la demencia vascular y la Enfermedad de Parkinson. El progresivo aumento de la esperanza de vida de la población hace que la demencia se haya convertido en uno de los mayores problemas de salud, afectando a una gran parte de la población mundial, especialmente a los ancianos, ya que constituye una epidemia silenciosa, siendo el estadio más avanzado del deterioro cognitivo. Todas las funciones sensoperceptivas, cognitivas, motoras y comportamentales pueden verse afectadas en los cuadros de demencia. (Portellano, 2005).

\section{Manifestaciones neuropsicológicas}

La evolución del deterioro neuropsicológico en la Enfermedad de Alzheimer es insidiosa y progresiva, afectando a memoria, lenguaje, praxias y gnosias. En las fases finales de la enfermedad se produce un síndrome afaso-apracto-agnósico generalizado.

\section{Trastornos de memoria}

El déficit de memoria se produce desde el comienzo de la enfermedad, interfiriendo de modo significativo en las actividades cotidianas del paciente. Lo más significativo es el comienzo de la enfermedad con alteraciones en la memoria inmediata y en la memoria de fijación, bajo la forma de olvidos (ej.: llaves, documentos, comida en el fuego, etc.), así como la dificultad para el aprendizaje de nuevas palabras y sucesos a pesar de la presentación repetida de estímulos. Se presentan también trastornos de memoria episódica con gradiente temporal, de tal forma que se recuerdan con mayor facilidad los acontecimientos más 
antiguos de la vida del paciente, pero no los que han sucedido más recientemente. La memoria semántica así como la memoria de procedimiento suelen estar conservadas hasta fases muy avanzadas de la enfermedad. (Portellano, 2005).

\section{Trastornos del lenguaje}

Las alteraciones del lenguaje suelen ser posteriores a los trastornos de memoria. En las fases iniciales de la enfermedad es frecuente la anomia, con dificultad para atribuir nombre a los objetos o personas conocidas. El lenguaje se empobrece paulatinamente, pero conserva su estructura fonológica y sintáctica. A medida que avanza la enfermedad se altera la estructura simbólica del lenguaje, con manifestaciones de Afasia transcortical o sensorial y otras alteraciones como ecolalia y palilalia. (Portellano, 2005).

\section{Evolución de la enfermedad}

La duración media de la enfermedad, hasta el fallecimiento, va en aumento, estimándose en doce años en los países desarrollados. En algunos casos, durante las primeras fases se acompaña de trastornos psíquicos como depresión y ansiedad. Las alteraciones de comportamiento son progresivas y terminan por producir una interferencia grave en la realización de las tareas diarias, originándose una gran discapacidad y una dependencia total frente al cuidador.

\section{Rehabilitación cognitiva de las demencias}

\section{Estimulación cognitiva}

La estimulación cognitiva (EC) es un proceso individualizado, adaptado a las necesidades cognitivas, emocionales y físicas de los pacientes, que busca rehabilitar funciones alteradas, a través de estímulos generados a partir de la neuropsicología que permitan generar procesos de neuroplasticidad (Arroyo, 2002).

El primer paso antes de diseñar un programa de EC es la realización de una com- pleta evaluación neuropsicológica con el fin de determinar aquellas capacidades preservadas y/o deterioradas. Los tratamientos de EC tienen como objetivo enlentecer el deterioro del paciente, adaptar sus habilidades cognitivas y mejorar su estado funcional y calidad de vida, así como la de su familia (Arroyo, 2002). Algunas de las características de estos tratamientos consisten en que pueden realizarse de forma individual o en grupo, se enfocan en las áreas de funcionamiento cognitivo preservadas, no tienen una limitación temporal, tienen en cuenta la fase o estadio de la enfermedad, los ejercicios pueden adecuarse al tipo específico de demencia y usualmente se incluye a la familia o al cuidador principal como parte del proceso. (Arrollo-Anlló, 2002)

La rehabilitación cognitiva de los ancianos con demencia tiene peculiaridades propias, ya que se trata de detener -en la medida de lo posible- un deterioro cognitivo que tiende a ser progresivo. Existen diversas técnicas utilizadas para la rehabilitación cognitiva en ancianos:

a) Terapia de orientación a la realidad Consiste en proporcionar al paciente con demencia una serie de claves personales al inicio de cada sesión para facilitar su orientación y prevenir el deterioro. Se le pregunta su nombre, edad, el lugar y fecha de nacimiento y diversas cuestiones que le permitan la ubicación espacio-temporal. La orientación en el tiempo consiste en preguntarles la fecha exacta (mes, año, estación del año y día de la semana) y la orientación en el espacio consiste en que identifiquen el lugar dónde se encuentran (edificio, dirección, ciudad, departamento y país).

\section{b) Técnica de la reminiscencia}

Permite evocar situaciones del pasado a través de un estímulo que puede ser un objeto antiguo, una fotografía, una música o una situación creada a través de los pro- 
pios pacientes mediante su escenificación. Se pueden utilizar objetos convencionales para generar una sesión, conversando en torno a un objeto próximo a los pacientes: un cuadro, una plancha, o un molinillo de café, evocando así recuerdos lejanos. Para realizar este tipo de talleres es imprescindible conocer los hábitos y los gustos de los pacientes en su juventud: si han crecido en un medio rural o urbano, que tipo de actividad laboral han desarrollado y a qué entorno cultural pertenecen. De este modo se van desarrollando diferentes actividades relacionadas con actividades profesionales, fiestas tradicionales, aperos y herramientas, bailes populares y vestidos regionales, comidas típicas, recetas de cocina, etc. La utilización del álbum de fotografías familiar puede ser de gran utilidad ya que facilita la reminiscencia de la memoria terciaria y tiene un valor emocional que produce un refuerzo intrínseco en el sujeto.

\section{c) Psicomotricidad vivenciada}

La psicomotricidad puede ser de gran utilidad en el contexto de la rehabilitación cognitiva de las demencias, especialmente la de tipo vivenciado, ya que combina activación cognitiva, sentido lúdico y rehabilitación motora. Los talleres de psicomotricidad son muy bien tolerados por la mayoría de los ancianos y proporcionan sentimientos de bienestar, al tiempo que constituyen un valioso instrumento para la rehabilitación cognitiva. Además, se trabajan otras áreas cognitivas: esquema corporal, orientación espacial, sentido del ritmo, atención, visopercepción, etc. La utilización de bailes, música y canciones hacen recordar y por tanto revivir el pasado del paciente, ligado a su memoria emocional y a experiencias personales importantes. Otra ventaja que ofrece la psicomotricidad en los ancianos es el mantenimiento de la función motora, especialmente en los enfermos de Alzheimer, que hasta estadios muy avanzados de la enfermedad man- tienen la funcionalidad. En los talleres de psicomotricidad se realizan ejercicios gimnásticos y rítmicos y los pacientes asocian el ejercicio físico al concepto de salud y bienestar, por lo que se sienten fácilmente motivados.

\section{d) Grupos de estimulación cognitiva}

Pretenden retrasar el proceso de deterioro cognitivo de los ancianos con demencia, siendo especialmente aconsejables para el tratamiento del Alzheimer en grado ligero o moderado.

Los grupos de estimulación se plantean mejorar la calidad de vida de los enfermos y la de sus cuidadores y tienen varios objetivos:

- Estimular a los pacientes para que mantengan sus facultades cognitivas y su actividad funcional.

- Activar su estilo de vida, evitando el aislamiento y la pasividad, facilitando su relación con otras personas en un contexto lúdico.

- Ayudar a los familiares aportándoles información, asesoramiento, modos de afrontamiento, pautas de actuación en el entorno familiar y apoyo psicológico.

\section{Se trabaja en tres áreas diferenciadas:} - Área Cognitiva: se trata de estimular al enfermo para que mantenga activadas sus funciones cognitivas: memoria, lenguaje, orientación, cálculo... Se utilizan técnicas de reminiscencia, psicomotricidad y orientación a la realidad, así como otras de tipo complementario como laborterapia o musicoterapia.

- Área Psicológica. Su objetivo es facilitar la ayuda psicológica al enfermo con demencia a través de la acción de grupo, permitiendo que se sienta integrado en un entorno que permite compartir preocupaciones, afrontando las limitaciones que causa el deterioro y aceptar la enfermedad. También se pretende prevenir y tratar las alteraciones comportamentales derivadas de la enfermedad, especialmente depre- 
sión, ansiedad, agitación o agresividad. Por último, a través de los grupos es posible incorporar estrategias y hábitos saludables en la vida del enfermo para favorecer el desarrollo de su estilo de vida.

- Área Social. Permite estimular su comunicación con el entorno, a través de actividades socioculturales, de ocio y de tiempo libre mediante excursiones, visitas a exposiciones, cine, teatro, etc. Estas actividades facilitan la cohesión entre los miembros del grupo.

\section{e) Actividades de rehabilitación cogni- tiva en el domicilio}

En el domicilio del paciente con demencia se pueden realizar algunas de las técnicas de orientación a la realidad, reminiscencia o psicomotricidad, así como otras, en función de su idiosincrasia y grado de deterioro. Estas actividades las puede realizar el cuidador u otras personas de la familia. Si el paciente solía jugar a las cartas o a otros juegos de mesa, es bueno continuar este hábito, compartiéndolo con los familiares. También hay otras actividades que se pueden compartir con cuidadores y familiares: la pelota, ver fotografías, construir puzzles o cuidar el jardín. Es mejor utilizar actividades que el paciente realizaba con anterioridad y que eran de su agrado.

Desde hace algún tiempo han empezado a aparecer diversos métodos de rehabilitación cognitiva de las demencias. Recientemente se ha editado en España El Baúl de los Recuerdos (Losada, 2004). Se trata de un método de estimulación cognitiva para

\section{CONCLUSIONES}

De lo expuesto en este trabajo queda claro que la neuroplasticidad tiene un efecto positivo en cuanto al tratamiento de enfermedades neurodegenerativas que evita el deterioro cognitivo produciendo mecanismos de plasticidad compensatoria, que enfermos de Alzheimer en el ambiente familiar, que pretende ayudar tanto al enfermo como a sus cuidadores. Ofrece un conjunto de elementos de estimulación, de un modo sencillo y práctico mostrando a los familiares cómo utilizar diferentes procedimientos y actividades cognitivas para mantener mentalmente activo al enfermo, estimulando las capacidades cognitivas que aún conserva. Consta de un manual de aplicación y 7 tomos, en los que se trabajan: atención y percepción, orientación, memoria, lenguaje, razonamiento y cálculo, música, motricidad y manualidades, y actividades de la vida diaria. Otra herramienta aparecida en el mercado español con la misma finalidad es el Taller de Memoria (Maroto, 2004).

En paralelo a la estimulación cognitiva en el domicilio se debe realizar una programación de pautas de vida saludables para evitar el progreso del deterioro, teniendo en cuenta las siguientes premisas:

a) Inclusión de ejercicios planificados y estructurados que impliquen activación psicomotora. (Maroto, 2004)

b) Disminuir al máximo los cambios en el entorno del paciente para evitar respuestas desadaptativas.

c) Incorporar la actividad física como un elemento imprescindible en el estilo de vida del paciente.

d) Estructurar un horario estable de actividades y rutinas diarias.

e) Evitar los episodios de sueño durante el día.

permite la recuperación de lesiones que puedan darse en un momento determinado.

Gracias a las últimas investigaciones realizadas sobre neuroplasticidad resulta evidente que el enfoque terapéutico orientado a estimularla constituye una buena 
alternativa para combatir demencias de una manera saludable ya que solo es necesario un buen acompañamiento de parte del equipo multidisciplinario, la familia y la voluntad del paciente.

La enfermedad de Alzheimer es una enfermedad cerebral que ocasiona problemas relacionados con la memoria, el pensamiento y el comportamiento y el tipo de demencia más común en las personas según varias estadísticas realizadas en las investigaciones, el 70-75\% de los casos corresponde a esta patología. En esta enfermedad podemos observar el deterioro cognitivo a través del tiempo, sin embargo gracias a los tratamientos físicos, neurológicos y neuropsicológicos es posible evitar a tiempo el progreso de la enfermedad.

La estimulación cognitiva (EC) es uno de estos tratamientos, un proceso individualizado, adaptado a las necesidades cognitivas, emocionales y físicas de los pacientes, que hace posible la generación de procesos de neuroplasticidad, a través de estímulos generados a partir de la neuropsicología y que con ello busca rehabilitar funciones alteradas.

Para ello es necesario la evaluación física como también cognitiva del paciente para determinar aquellas capacidades preservadas y/o deterioradas. Luego se trabajan en áreas diferenciadas (cognitiva, psicológica y social), con el fin de cumplir con el objetivo enlentecer el deterioro del paciente, adaptar sus habilidades cognitivas y mejorar su estado funcional y calidad de vida, así como la de su familia.

\section{REFERENCIAS}

1. Arrollo-Anlló, E. M. (2002). Estimulación Psicocognoscitiva en las demencias. Barcelona : Proas Science.

2. Arthur F. Kramer, S. H. (1999). Envejecimiento, fitness y función neurocognitiva . 400,418-419.

3. Association, A. (2016). Información Básica sobre la enfermedad del Alzheimer . Alzheimer's Association.

4. Hernández Muela S, M. F. (2004). Plasticidad Nueronal Funcional. Rev. Neurol.

5. Horton, A. M. (1997). The Neuropsychology Handbook. Foundations and Assessment. New York: Springer Publications Corporation.

6. Losada, D. (2003). Bául de los Recuerdos. Manual de Estimulación cognitiva para enfermos de Alzheimer en ambiente familiar. Madrid: TEA Ediciones.

7. Maroto, M. A. (2004). Taller de
Memoria . Madrid: TEA Ediciones .

8. Nieto Sampedro, M. (2003). Plasticidad Neuronal. Mente y Cerebro, 72-80.

9. Peña, B. (07 de Octubre de 2019). Muy Salud. Recuperado el 15 de Agosto de 2020, de https://muysalud.com/mente/ demencias-tipos-caracteristicas-y-sintomas/

10. Portellano, J. A. (2005). Introducción a la Neuropsicología. Madrid : McGRAW-HILL/INTERAMERICANA DE ESPAÑA, S.A.U. 